\title{
Das Gespenst des Kapitals
}

\author{
S. Schipper \\ Institut für Humangeographie, Goethe-Universität Frankfurt, Campus Westend - PEG, \\ Grüneburgplatz 1, 60323 Frankfurt, Germany \\ Correspondence to: S. Schipper (s.schipper@geo.uni-frankfurt.de)
}

Vogl, J.: Das Gespenst des Kapitals, 4. Aufl., Zürich, Diaphanes, 224 pp., ISBN-13: 978-3-03734-116-2, €14.90, 2011.

Seit Beginn der jüngsten Wirtschafts- und Finanzkrise sind in den kritischen Sozialwissenschaften zahlreiche Veröffentlichungen erschienen, die sich aus marxistischer, regulationstheoretischer oder poststrukturalistischer Perspektive den gesellschaftlichen Ursachen selbiger widmen. Mit seinem sprachgewaltigen Essay „Das Gespenst des Kapitals“ liefert der Berliner Literaturwissenschaftler Joseph Vogl dazu einen weiteren lesenswerten Beitrag, indem er in sechs Kapiteln genealogisch die Entstehung ökonomischer „Glaubenssätze“ (S. 29) von der politischen Ökonomie des 17. Jh. bis zum Neoliberalismus des späten 20. Jh. nachzeichnet. Ausgehend von der Annahme, ,dass das ökonomische Wissen der letzten dreihundert Jahre" performativ ,die wirtschaftlichen Tatsachen geschaffen hat, mit deren Entzifferung es sich selbst konfrontiert" (S. 8), fragt Vogl nach der Konsistenz jener grundlegenden ökonomischen Paradigmen, welche das Marktgeschehen als Schauplatz von Ordnung, Ausgleich und gesellschaftlicher Vernunft repräsentieren und wonach folglich sämtliche ,Zweckwidrigkeiten, Übel und Pannen im System mit dessen weiser Einrichtung vereinbar" (S. 29) zu sein haben. Zum Vorschein kommen dabei ernsthafte Zweifel, ob zum einen das kapitalistische System tatsächlich rational und effizient arbeitet und ob zum anderen in der neoklassischen Wirtschaftstheorie überhaupt ein plausibles finanzökonomisches Narrativ existiert.

Ausgangspunkt seines Unterfangens, die Herausbildung des klassisch-neoklassischen ökonomischen Diskurses zu rekonstruieren, sind die elementaren, im 17. Jh. entstehenden Gründungsfiguren der politischen Ökonomie, wonach ausschließlich der Markt als Garant spontaner Ordnung funktionieren könne, die segensreiche Wirkung von Wettbewerb den Wohlstand Aller mehre sowie Angebot und Nachfrage automatisch zu Gleichgewichten tendierten. Zurück führt Vogl diese Figuren auf die Erfindung des ökonomischen Men- schen in naturrechtlichen und moralphilosophischen Diskursen jener Zeit, in denen erstmalig anthropologisch unterstellt worden sei, dass sich Gesellschaft über das rationale Agieren von selbstsüchtigen Individuen erklären und verstehen lasse. In Anlehnung an das Erkenntnismodell empirischer Naturerfahrungen sei so letztlich eine Wissenschaft der Ökonomie geboren worden, welche die Komplexität sozialer Beziehungen auf eine kalkulierbare Mechanik atomisierter Interessen reduziere und diese mittels mathematischer Berechnungen zu begreifen trachte. Zugrunde liege der ökonomischen Wissensproduktion somit seit ihrer Gründung ein anthropologischer Naturalismus, wonach die unsichtbare Hand des Wettbewerbs gleichsam ein Naturgesetz exekutiere und der Markt als privilegierter Mechanismus sozialer Ordnung erscheine. Implizit sei daher bereits den elementaren Gründungsfiguren der politischen Ökonomie die Aufforderung, Marktgesetze nicht nur mathematisch zu beschreiben, sondern die Idee des Wettbewerbs auch politisch wahr zu machen und vor dem Unheil staatlicher Eingriffe zu bewahren. Indem das Wahrheitsprogramm um Markt und Wettbewerb als geschichtliches Ziel ,eine ebenso aktive wie resolute Politik verlangt“" (S. 58), habe es in den letzten 300 Jahren performativ eine ökonomische und soziale Welt hervorgebracht, welche es stets vorgab, lediglich deskriptiv zu erfassen. Die Theorie freier Märkte habe sich also fortdauernd mit dem theoretischen Wunschdenken beschäftigt, dem „Marktgeschehen eine vorbildliche Ordnungsleistung zu attestieren und damit einen Standard zu schaffen, der besser als jedes andere Modell das Maß für eine harmonische Gesellschaftsordnung bereitstellen" (S. 51) könne. Bezogen auf die Gegenwart kommt Vogl daher zu dem Schluss, dass die Fabel der ausgleichenden Kräfte des Marktes, die bereits Gestalt annahm, „,bevor der Markt zu funktionieren begann“ ein „privilegiertes Bildreservoir“ (S. 55) für die Selbstrepräsentation moderner Gesellschaften hervorgebracht habe. Möglich geworden sei diese privilegierte Stellung, da es der Lehre von der Ökonomie erfolgreich gelungen sei, sich als systematische Wissenschaft der angewandten Mathematik zu 
präsentieren. Einer solchen Mathematisierung sozialer Beziehungen liege jedoch laut Vogl eine genauso fundamentale wie problematische Annahme zugrunde: Unabhängig davon, ob Markt und Wettbewerb im realen Wirtschaftsgeschehen tatsächlichen stets zu einem Gleichgewicht tendierten, seien derartige Gleichgewichtspostulate vor allem eine paradigmatische Bedingung und logische Notwendigkeit, um überhaupt die „ökonomischer Theorie als Theorie“ (S. 61) und als mathematische Wissenschaft begründen zu können.

Nichtsdestotrotz seien die ökonomischen Glaubenssätze jedoch bereits ab dem Ende des 18. Jh. ins Wanken geraten, da mit der Entstehung von ungedecktem Papiergeld, der Ausweitung des Kreditverkehrs und dem Aufkommen von Fragen der Finanzökonomie ,die notorischen Gleichgewichtsmodelle der politischen Ökonomie ihr verlässliches, gleichsam naturwüchsiges Statut" (S. 82) zu verlieren drohten. Mit dem Zusammenbruch des Bretton Woods Systems im Jahr 1973, dem Sprung in die Nichtkonvertierbarkeit von Währungen und dem endgültigen Übergang von Waren- zu Kreditgeld habe sich nach Vogl dieses latente Unbehagen zu einer neuen Herausforderung für die ökonomische Theorie gewandelt. Denn zu beweisen galt es nun, dass sich auch und „gerade im freien Spiel der Finanzmärkte die ordnende Vernunft von Marktbeziehungen" (S. 88) offenbare. Vor diesem Hintergrund ließe sich als wesentlicher Fluchtpunkt sämtlicher theoretischer, institutioneller und technischer Finanzmarktinnovationen ab den 1970er Jahren der Versuch ausmachen, ein stabiles Währungssystem nicht mehr durch feste Devisenkurse oder eine Golddeckung, sondern durch Marktmechanismen selbst zu garantieren. Es galt also bezüglich Währungsrisiken Mechanismen zu entwickeln und zu etablieren, die dafür Sorge tragen, dass die Kosten der Risiken von den Marktsubjekten selbst, etwa durch Derivate und Termingeschäfte, getragen werden - wodurch staatliche Regulierung letztlich als ineffizient und unnötig erscheint. Mit der Einführung flexibler und deregulierter Wechselkursregime sei demnach der Finanzmarkt schließlich symbolisch zum $\mathrm{Ma} ß$ aller Märkte bzw. zum Modell des Marktgeschehens überhaupt avanciert. Denn da auf Finanzmärkten scheinbar alle Bedingungen der Effizienzmarkthypothese gegeben sind (vollkommener Wettbewerb, keine Transaktionskosten, vollständige Informationen) und die Preise sämtliche Informationen reflektieren, habe die neoklassische Finanzökonomie in ihren Modellen theoretisch begründen können, dass freie Märkte für immer Ungleichgewichte und Krisen ausschließen. Komplementiert worden sei dieser Siegeszug ökonomischer Glaubenssätze auf dem Feld der Finanzmärkte durch eine parallele Genese neoliberaler Schulen, welche die Grenzen des Ökonomischen systematisch überschritten hätten, indem sie einem ökonomischen Imperialismus folgend tendenziell sämtliche Lebensbereiche unter die Marktlogik zu subsumieren trachten - etwa durch die Verallgemeinerung der Unternehmensform und die Auflösung des Widerspruchs zwischen Arbeit und Kapital im Begriff des Humankapitals.
Mit seiner Genealogie ökonomischer Glaubenssätze von der politischen Ökonomie des 17. Jh. über die Entstehung deregulierter Finanzmärkte bis hin zur neoliberalen Humankapitaltheorie hat Vogl allerdings keine evolutionäre Dystopie einer unentrinnbaren Ökonomisierung aller gesellschaftlichen Beziehungen formuliert. Im Schlusskapitel kann er vielmehr überzeugend zahlreiche „Leckstellen“ (S. 141) und Widersprüche aufzeigen, welche darauf hindeuten, dass sich das soziale Gewebe kaum „,mit der Einpflanzung neoliberaler Systemideen“ (S. 141) vollständig durchwalten lässt. Denn zum einen hätten die wiederkehrenden Finanzkrisen der letzten Jahrzehnte offenbart, dass sich die kapitalistische Ökonomie keineswegs so verhält, wie sie sich gemäß ihrer wirtschaftswissenschaftlichen Apologeten verhalten sollte. Statt Ausnahmefälle zu sein, gehörten Krisen - und zwar gerade auf scheinbar perfekten Finanzmärkten - vielmehr zum regulären Funktionsablauf und dokumentierten daher zugleich auch ,eine Krise jenes Theorieprofils“, welches stets die unschlagbare ,innere Stabilisierungstendenz der Finanzmärkte" (S. 150) postuliert hat. Zum anderen kann Vogl durch die Rekonstruktion der Genese der ökonomischen Glaubenssätze und der damit verbundenen Finanzinstitutionen deutlich machen, dass kapitalistische Gesellschaften als Ausdruck einer Kette kontingenter Ereignisse zu betrachten sind. Frei nach Marx machen Menschen also nicht nur ,ihre eigene Geschichte", sondern produzieren auch ihre eigenen Finanzmärkte, aber eben ,nicht aus freien Stücken, nicht unter selbstgewählten, sondern unter unmittelbar vorgefundenen, gegebenen und überlieferten Umständen“ (MEW 8, S. 115). Durch die Denaturalisierung des dominanten ökonomischen Wissens - immerhin ein nicht unwesentlicher Bestandteil dieser ,vorgefundenen, gegebenen und überlieferten Umstände“ - und durch die Feststellung, dass „Finanzmärkte als Märkte aller Märkte so operieren, dass sie mit rationalen Entscheidungsprozessen systematisch Unvernunft produzieren“" (S. 174), hat Vogl ein fundiertes Plädoyer für einen radikalen politischen Kurswechsels formuliert, die gesellschaftliche Abhängigkeit von Märkten und deren Krisen zu reduzieren. Für eine kritische Geographie und Gesellschaftswissenschaft folgt daraus, die „verheerenden logischen Löcher“ der kapitalistischen Glaubenssätze als Voraussetzung zu nutzen, um ,anormale Verläufe und systemische Instabilität als Resultat des normalen Funktionierens kapitalistischer Ökonomie“ (S. 154) zu begreifen und an der „Konstitution eines alternativen Wissens vom kapitalistischen Prozess“ (S. 153f) mitzuwirken. 